\title{
Fractional-Order Control of USV Course-Keeping Based on Self- Adaptive Weight PSO Algorithm
}

\author{
Guang-yu Li ${ }^{1,2}$, Chen Guo ${ }^{1}$, Yan-xin $\mathrm{Li}^{2}$ and Wu Deng* ${ }^{2,3,4}$ \\ ${ }^{1}$ Information Science and Technology College of Dalian Maritime University, Dalian \\ 116026, China \\ ${ }^{2}$ Software College of Dalian Jiaotong University, Dalian 116052, China \\ ${ }^{3}$ Guangxi Key Laboratory of Hybrid Computation and IC Design Analysis, Guangxi \\ University for Nationalities, Nanning 530006 China \\ ${ }^{4}$ The Provincial Key Laboratory for Computer Information Processing Technology, \\ Soochow University, Suzhou 215006 \\ ${ }^{1}$ ligyu@163.com, ${ }^{2}$ dmuguoc@126.com, ${ }^{2}$ lyx7977@163.com, ${ }^{2}$ dw7689@gmail.com
}

\begin{abstract}
In order to improve the stability of underactuated surface vessels (USV) coursekeeping, a fractional-order $P I^{\lambda} D^{\mu}$ controller based on self-adaptive weight particle swarm optimization (SAWPSO-FOCPID) was proposed for ship autopilot and had good overall control ability. The integral-order $\lambda$ and differential-order $\mu$ can improve the controller with more robustness and take on stronger disturbance rejection ability. However, the difficulties for parameter verification are increased. A new designing method of $P I^{\lambda} D^{\mu}$ controller based on SAWPSO algorithm was proposed in order to balance the global search ability and local search ability of PSO algorithm. And at the same time, the difficulties of tuning more parameters and designing complexity of fractional-order $P I^{\lambda} D^{\mu}$ controller were solved. The SAWPSO-FOCPID controller was compared with the traditional PSO-PID controller under the same condition. The simulation results showed that the SAWPSO-FOCPID controller has a high dynamic tracking performance, small overshoot and strong immunity.
\end{abstract}

Keywords: Underactuated surface vessels, Course-keeping control, Self-adaptive weight particle swarm optimization algorithm, Fractional-order $\mathrm{PI}^{\lambda} D^{\mu}$ controller, Automatic rudder

\section{Introduction}

The underactuated system is defined as a system that the spatial dimension of control input is less than spatial dimension of configuration, which is the number of control system input less than the freedom degrees of system [1]. The underactuated surface vessel is a large inertia, nonlinear and time-delay system. Additionally, when the ship is sailing, by uncertain wind wave and current of the marine environment impact, it gradually deviates from a predetermined course. Therefore ship course control is an important criterion to judge the quality of ship maneuverability, the performance of ship course control relates to safety and economic of ships navigation. When the ship is sailing, the disturbance is very complicated. To ensure the ship sail in accordance with the expected course, the course controller must control automatic rudder to correct the course deviation due to the disturbance. In order to obtain satisfactory control effect, many scholars made different attempts in the area of autopilot steering control.

The traditional course control device of USV is the digital PID automatic rudder that is sensitive to the high-frequency disturbances, and leads to frequent steer. In recent 
years, artificial intelligence methods are used for tuning PID parameters, and the control precision of ships course is effectively improved. Fu et al., [2] made the least squares support vector machine (LSSVM) apply to the modeling identification of the ship course movement, and designed a ship course complex inverse control scheme based on LSSVM and PID. Wang et al., [3] used dynamic neural fuzzy model (DNFM) based adaptive control algorithm for ship course control, and overcome uncertainties arising from changes of model parameters. In this paper, for ship course autopilot, the fractional-order $P I^{\lambda} D^{\mu}$ controller was designed, and it had a flexible structure, strong robustness and disturbance rejection.

The fractional-order $P I^{\lambda} D^{\mu}$ controller was proposed by Podlubny $\mathrm{I}$ in 1999. Compared with traditional PID, the integral-order $\lambda$ and differential -order $\mu$ make the controller have more than two adjustable parameters that make it more flexible, more robust [4-5] .But at the same time, as a result of more than two adjustable parameters, it is more difficult to design fractional-order controller [6-9]. Thus, the parameter tuning method of the fractional-order control system has become a hot topic. Yao et al., [10] proposed a design method for robust fractional order PID control system based on particle swarm optimization that enhance the robustness and response speed of the synchronous machine excitate regulator. Zhang et al., [11] used fuzzy fractional synovial theory in the parameter tuning of permanent magnet synchronous motor controller.

Hence, this study presented a self-adaptive weight particle swarm optimization which solved the premature question of PSO and easy to produce oscillations in the vicinity of the global optimal solution in late algorithm. At the same time, the questions of controller more tuning parameters and design complexity were solved, and the static and dynamic performance of the system were improved. Compared with fractional-order $P I^{\lambda} D^{\mu}$ controller based on SAWPSO and integer-order PID controller based on PSO, the controller based on SAWPSO is superior to the conventional PSO-PID controller in the ability of immunity and the adaptive ability of control object parameters change.

\section{Mathematical Model of Underactuated Surface Vessels}

\subsection{Maneuvering Mathematical Model}

For an ocean vessel moving in 6 DOF, the 6 different motion components respectively represent the position and direction. The first three coordinates $(x, y, z)$ and first-order differential denote ship position and translational motion along $\mathrm{x}, \mathrm{y}$ and $\mathrm{z}$ axes. The last three coordinates $(\phi, \theta, \psi)$ and first-order differential denote ship direction and rotational motion. The 6 different motion components respectively are conveniently denoted as surge, sway, heave, roll, pitch, and yaw [12], see Figure 1.

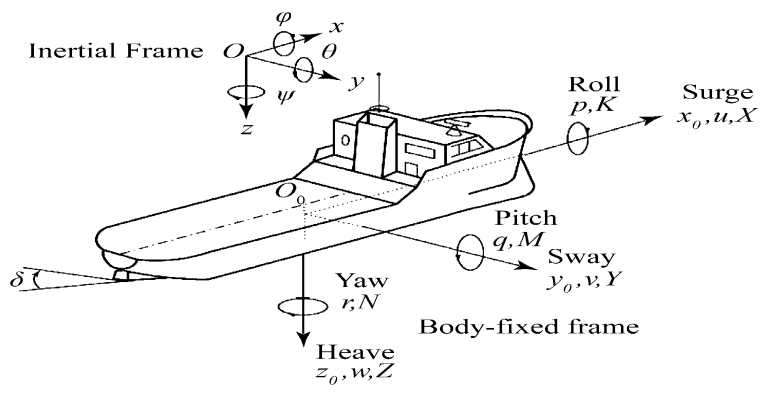

Figure 1. Reference Frames and Variables for Ship Motion Description 
Considering the course control problem of an underactuated surface vessel, the vessel is moving in the horizontal plane, and the heave, roll, and pitch are usually neglected

The mathematical model [13-14] of an underactuated surface vessel can be written as below

$$
\begin{aligned}
& \left\{\begin{array}{l}
\dot{x}=u \cos \psi-v \sin \psi \\
\dot{y}=u \sin \psi+v \cos \psi \\
\dot{\psi}=r
\end{array}\right. \\
& \left\{\begin{array}{l}
\dot{u}=\frac{m_{22}}{m_{11}} v r-\frac{d_{11}}{m_{11}} u+\frac{1}{m_{11}} \tau_{u} \\
\dot{v}=-\frac{m_{11}}{m_{22}} u r-\frac{d_{22}}{m_{22}} v \\
\dot{r}=\frac{m_{11}-m_{22}}{m_{33}} u v-\frac{d_{33}}{m_{33}} r+\frac{1}{m_{33}} \tau_{r}
\end{array}\right.
\end{aligned}
$$

where $x, y$ and $\psi$ denote the surge displacement, sway displacement, and yaw angle in the earth fixed frame; $u, v$ and $r$ are the surge, sway and yaw velocities, respectively; $m_{11}, m_{22}$ and $m_{33}$ denote the inherent mass and additional mass of ship, which are model uncertainties; $d_{11}, d_{22}$ and $d_{33}$ denote the hydrodynamic damping of the surge displacement, sway displacement, and yaw angle, which are model uncertainties; $\tau_{u}$ and $\tau_{r}$ denote longitudinal force and torque of turning ship.

\subsection{Norrbin Nonlinear Model for Course Control}

The USV usually looks as a dynamic system in the design of ship course controller. The system input is rudder angle $\delta$, output is yaw angle $\psi$. In the case of steering is not very frequent, the Norrbin nonlinear model of course control for USV is deduced from the maneuvering mathematical model of USV.

$$
\ddot{\psi}=-a_{1} \dot{\psi}-a_{2} \psi^{3}+b \delta
$$

In which, $a_{1}=\alpha b, a_{2}=\beta b, b=K / T$ are all model parameters. $K$ and $T$ are ship indexes; $\alpha$ and $\beta$ are nonlinear coefficient. $K, T, \alpha$ and $\beta$ are relevant to the speed and structure of ship.

\section{Fractional Calculus and Fractional-Order Controllers}

\subsection{Fractional Calculus Definition}

There are three kinds of fractional calculus definition. Fractional calculus is a theory of arbitrary order differential and integral, and it is the promotion definition of integer-order calculus. Its basic operation operator is as follows 


$$
{ }_{a} D_{t}^{\alpha}=\left\{\begin{array}{l}
\frac{d^{\alpha}}{d t^{\alpha}}, \operatorname{Re}(\alpha)>0 \\
1, \operatorname{Re}(\alpha)>0 \\
\int_{\alpha}^{t}(d \tau)^{-\alpha}, \operatorname{Re}(\alpha)<0
\end{array}\right.
$$

In which, $a$ and $t$ are upper and lower limit of operation operator, $\alpha$ is calculus order and may be any complex number plural, then it is assumed to be real numbers[15].

In the development of fractional calculus theory, there are many kinds of fractional calculus definitions, such as Grunwald-Letnikov definition, Riemann-Liouviblle definition and Caputo definition.

The Grunwald-Letnikov formula:

$$
{ }_{a} D_{t}^{\alpha} f(t)=\lim _{h \rightarrow 0} \frac{1}{h^{\alpha}} \sum_{i=0}^{\left[\frac{t-a}{h}\right]}(-1)^{j}\left(\begin{array}{l}
\alpha \\
j
\end{array}\right) f(t-j h)
$$

In which, $[x]$ denotes $x$ integer, $\quad$ is binomial coefficient.

$$
\left(\begin{array}{l}
\alpha \\
j
\end{array}\right)
$$

The Riemann-Liouviblle formula:

$$
{ }_{a} D_{t}^{\alpha} f(t)=\frac{1}{\Gamma(m-\alpha)}\left(\frac{d}{d t}\right)^{m} \int_{a}^{t} \frac{f(\tau)}{(t-\tau)^{1-(m-\alpha)}} d \tau
$$

In which, $\Gamma(\bullet)$ is Gamma function.

The Caputo formula:

$$
{ }_{a} D_{t}^{\alpha} f(t)=\frac{1}{\Gamma(m-\alpha)} \int_{a}^{t} \frac{f^{m}(\tau)}{(t-\tau)^{1-(m-\alpha)}} d \tau
$$

In which, $m-1<\alpha<m, m \in N$.

From the definition of three kinds of fractional-order calculus can be seen, it is different in the fractional-order differential and integer-order differential of continuous function at a certain point. It is not for extremum at this point, but it is associated with the initial moment of all time to the point before the function value, and it has memory.

\subsection{Fractional-Order $P I^{\lambda} D^{\mu}$ Controller}

To improve the control accuracy, based on conventional PID controller, the integral-order $\lambda$ and differential-order $\mu$ are extended to real domain, and $P I^{\lambda} D^{\mu}$ controller is obtained, its differential equation expression is

$$
u(t)=K_{p} e(t)+K_{i} D_{t}^{-\lambda} e(t)+K_{d} D_{t}^{\mu} e(t)
$$

In which, $D_{t}^{\alpha} \equiv{ }_{a}^{C} D_{t}^{\alpha}$ is the Caputo definition, $\lambda>0$ and $\mu>0$ are any real number and the order of fractional-order controller. The Laplace transform of Caputo definition is

$$
L\left\{{ }_{a}^{C} D_{t}^{\alpha} f(t)\right\}=s^{\alpha} F(s)-\sum_{k=0}^{n-1} s^{\alpha-k-1} f^{(k)}(0)
$$

By Equation (8) (9), the transfer function of fractional-order $P I^{\lambda} D^{\mu}$ controller is 


$$
G_{c}(s)=K_{p}+\frac{K_{i}}{s^{\lambda}}+K_{d} s^{\mu}
$$

Because the order of differential $\mu$ and integral $\lambda$ is any positive real number, the integer-order PID controller is a special case of fractional-order $P I^{\lambda} D^{\mu}$ controller in $\lambda=1$ and $\mu=1$. When $\lambda=1$ and $\mu=0$, it is PI controller. When $\lambda=0$ and $\mu=1$, it is PD controller. The fractional-order $P I^{\lambda} D^{\mu}$ controller has more than two adjustable parameters; the system control performance can be improved by properly selecting integral -order $\lambda$ and differential-order $\mu$.

\subsection{Improved Approximation Oustaloup of Fractional-Order Calculus}

In order to make the method of integer-order controller apply to the fractionalorder controllers, it needs to become a fractional calculus as approximate integerorder calculus. In this paper, an improved Oustaloup approximation is applied. $s^{\alpha}$ is approximated as integer-order calculus, fitting frequency range is $\left(\omega_{b}, \omega_{h}\right)$,order is $N$. By this method, approximation integer-order transfer function is shown as

$$
s^{\alpha}=\left(\frac{d \omega_{b}}{b}\right)^{\alpha}\left(\frac{d s^{2}+b s \omega_{h}}{d(1-\alpha) s^{2}+b s \omega_{h}+d \alpha}\right)\left[\frac{1+\frac{s}{d \omega_{b} / b}}{1+\frac{s}{d \omega_{h} / b}}\right]^{\alpha}
$$

In which, $0<\alpha<1, b>0, d>0, d=j \omega$, the fractional part of formula (11) $K(s)$ expressed as zeros, poles form of a rational transfer function

$$
K(s)=\lim _{N \rightarrow \infty} K_{N}(s)=\lim _{N \rightarrow \infty} \prod_{k=-N}^{N} \frac{1+s / \omega_{k}^{\prime}}{1+s / \omega_{k}}
$$

The $k$ zero, pole is

$$
\begin{aligned}
& \omega_{k}^{\prime}=\omega_{b}\left(\frac{\omega_{h}}{\omega_{b}}\right)^{\frac{N+k+\frac{1}{2}(1-\alpha)}{2 N+1}} \\
& \omega_{k}=\omega_{b}\left(\frac{\omega_{h}}{\omega_{b}}\right)^{\frac{N+k+\frac{1}{2}(1+\alpha)}{2 N+1}}
\end{aligned}
$$

Construct the continuous rational transfer function model of fractional calculus operator:

$$
G(s)=K\left(\frac{d s^{2}+b \omega_{h} s}{d(1-\alpha) s^{2}+b \omega_{h} s+d \alpha}\right) \prod_{k=-N}^{N} \frac{1+s / \omega_{k}^{\prime}}{1+s / \omega_{k}}
$$

In which, $K=\left(\omega_{b}, \omega_{k}\right)$. 


\section{The Design of Fractional-Order $P I^{\lambda} D^{\mu}$ Controller Based On SAWPSO}

\subsection{SAWPSO Tuning Fractional-Order Controller Parameters}

The formula (10) shows that fractional-order $P I^{\lambda} D^{\mu}$ controller adds two adjustable parameters $\lambda$ and $\mu$. Therefore, the fractional-order $P I^{\lambda} D^{\mu}$ controller has five adjustable parameters that are $\left(K_{p}, K_{i}, K_{d}, \lambda, \mu\right)$. Through the reasonable choice of the five parameters, the more ideal control effect will be obtained, and the performance of the system will be effectively improved. But the design complexity and workload of controller will be increased. Therefore, in this paper, the SAWPSO algorithm was applied to the design of fractional-order $P I^{\lambda} D^{\mu}$ controller, and optimized the five parameters tuning of fractional-order $P I^{\lambda} D^{\mu}$ controller.

\subsection{Sawpso Algorithm}

The particle swarm optimization algorithm originates in the research of prey behavior of birds [16]. It is essentially belongs to the iterative random search algorithm, has a parallel processing feature, and good robustness, easy to implement. In principle, it can be used on the larger probability to find the global optimal solution of the optimal problem, and has high calculation efficiency. Because the particle of PSO gathers to its historical best position and the neighborhood or community historical best position. It makes the rapid convergence effect of the particle population, and prone to fall into the local extremum, premature convergence or stagnation [17-19].

At the same time, the performance of PSO is dependent on the parameters of the algorithm. The algorithm specific parameter settings are different, the results may vary. The algorithm specific parameters mainly depend on mainly depends on, inertia weight $\omega$, learning factor and increasing the compression factor etc.

In the PSO algorithm, supposes the total number of particles for $N$, each particle has a position $x_{i}$ in space, this particle flight forward from $x_{i}$ by the speed $v_{i}$, the optimal position which each particle search in the space is $p_{i}$, the optimal position which the particle swarm search in the space is $P_{g}$, the second iteration correction of $x_{i}$ is

$$
v_{i}^{k}=\left[v_{i 1}^{k}, v_{i 2}^{k}, \ldots, v_{i n}^{k}\right]
$$

Then

$$
v_{i}^{k}=\omega v_{i}^{k-1}+c_{1} \operatorname{rand}_{1}\left(p_{i}-x_{i}^{k-1}\right)+c_{2} \operatorname{rand}_{2}\left(p_{g}-x_{i}^{k-1}\right) ; x_{i}^{k}=x_{i}^{k-1}+v_{i}^{k-1}, i=1,2, \ldots, N
$$

In the formula (4), $k$ is the number of iterations; $c_{1}$ and $c_{2}$ are acceleration factor, which keep the motion inertial of particles and have the ability of extending search space trend; rand $_{1}$ and rand $_{2}$ are random number between 0 and 1; $\omega$ is inertia factor, the search ability can be changed by adjust inertia factor. The termination condition of the improved PSO algorithm is that the largest iterations number or the fitness value of the optimal position which particle swarm searches so far meets the predetermined minimum fitness threshold value.

In the PSO algorithm, the larger weight factor is conducive to jump out the local minimum point and facilitates global search, the smaller inertia factor is conducive to accurate local searching for the current search area and facilitates algorithm convergence. Therefore, for the questions of PSO algorithm easy to premature and oscillate in the near global optimal solution in the algorithm late. In this paper, a 
self-adaptive weight particle swarm optimization algorithm (SAWPSO) was applied, which balances the global search ability and the local improved ability of PSO algorithm. The nonlinear dynamic inertia weight coefficient formula was adopted as

$$
\omega=\left\{\begin{array}{l}
\omega_{\min }-\frac{\left(\omega_{\max }-\omega_{\min }\right) *\left(f-f_{\min }\right)}{f_{\text {avg }}-f_{\min }}, f \leq f_{\text {avg }} \\
\omega_{\max }, f>f_{\text {avg }}
\end{array}\right.
$$

In which, $\omega_{\max }$ and $\omega_{\min }$ are the maximum and minimum value of $\omega, f$ is the current objective function value of particle, $f_{\text {avg }}$ and $f_{\min }$ are the average target value and the minimum target value of current particle. Because of the inertia weight changed with objective function value of the particle automatically, it is called self-adaptive weight.

The SAWPSO algorithm steps are as follows:

(1) Initialize particle swarm, the position $x_{i}$ and speed $v_{i}$ of each particle are generated randomly;

(2) The position vector of each particle is used as the parameter of the $P I^{\lambda} D^{\mu}$ controller, the current adaptive values $=$ of each particle is calculated respectively, and initialize $P_{i}$ and $P_{g}$;

(3) Update individual extreme value. The adaptive value of each particle is evaluated, that is, the current adaptive value $P_{i}(x)$ of the first i particle contrasts with the individual extreme $P_{i}$ of this particle. If the former is excellent, then update $P_{i}$, otherwise keep it;

(4) Update global extreme value. Choose the best from all the particles $P_{i}$, and as the global extreme value $P_{g}$.

(5) Update speed and position. The speed ${ }^{v_{i}}$ and position ${ }^{x_{i}}$ of each particle are updated through formula (17).

(6) Check to see whether the termination condition is satisfied. If satisfied, then exit; otherwise, go to step (2).

\subsection{Objective Function Selection}

This paper used the SAWPSO algorithm to tune the parameters $\left(K_{p}, K_{i}, K_{d}, \lambda, \mu\right)$ of fractional-order $P I^{\lambda} D^{\mu}$ controller; the space dimension of solution was five and the improved Oustaloup filtering method was used. The optimization objective function was comprehensively considered through the error square integral and system overshoot of control loop, the formula is

$$
J=\omega_{1} \int_{0}^{+\infty} e^{2}(t) d t+\omega_{2} \sigma
$$

In which, $e(t)$ is the system error. $\sigma$ is the system overshoot. $\omega_{1}$ and $\omega_{2}$ are weights.

\section{Simulations}

The simulation experiment uses the data of 5446TEU container ship of COSCO Group, supposes the rated speed of this ship is $V=24.51 \mathrm{knots}$, and the parameters are shown in Table 1. 
Table 1. Parameters of 5445TEU Container Ship

\begin{tabular}{|c|c|c|c|}
\hline Parameter & Value & Parameter & Value \\
\hline $\begin{array}{l}\text { Ship length } \\
\text { LOA }\end{array}$ & $280 \mathrm{M}$ & Two column length L & $267 \mathrm{M}$ \\
\hline Ship width B & $39.8 \mathrm{M}$ & $\begin{array}{l}\text { gravity center } \\
\text { distance }{ }_{c}\end{array}$ & $2.64 \mathrm{M}$ \\
\hline Rudder area $A_{\delta}$ & $61.0 \mathrm{M} 2$ & Square coefficient ${ }^{C_{b}}$ & 0.67 \\
\hline No load mass $\mathrm{m}$ & 3.5453 Million tons & Fully load mass m & $\begin{array}{l}\text { 6.5531 Million } \\
\text { tons }\end{array}$ \\
\hline Design draft $T$ & $12.532 \mathrm{M}$ & Fully load draft & $14.023 \mathrm{M}$ \\
\hline
\end{tabular}

According to Table 1, the parameters can be calculated $K=0.2419, T=2067958$, $\alpha=11.6049$ and $\beta=10.966$ in the rated speed.

According to design steps of controller, the fitting frequency of Oustaloup filtering method $\left(\omega_{b}, \omega_{k}\right)$ is $[0.001,1000]$, take order $\mathrm{N}=5$. The total of particle swarm is 50 . The particle size is five that is $\left[K_{P}, K_{i}, K_{d}, \lambda, \mu\right], c_{1}=0.12$, $c_{2}=1.2$. The maximum inertia weight is 0.9 and the minimum inertia weight is 0.6 , and the number of iteration steps is 100 . Choosing fractional-order $P I^{\lambda} D^{\mu}$ controller based on SAWPSO and integer-order PID controller based on PSO, the maximum rudder angle limits at $-35^{\circ} \sim+35^{\circ}$, the expected course angle takes $25^{\circ}$ in $0 \sim 1000$ seconds. Under no wind and wave disturbance, the step response curves of two different control methods are shown in Figure2, Figure 3 and Figure4.

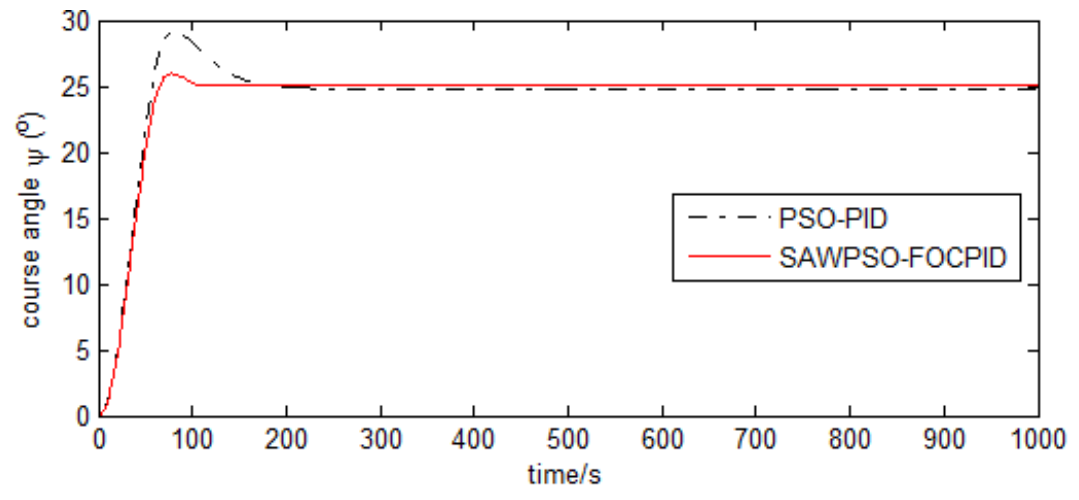

Figure 2. Ship Course Tracking Curve under No Wind and Wave Disturbance

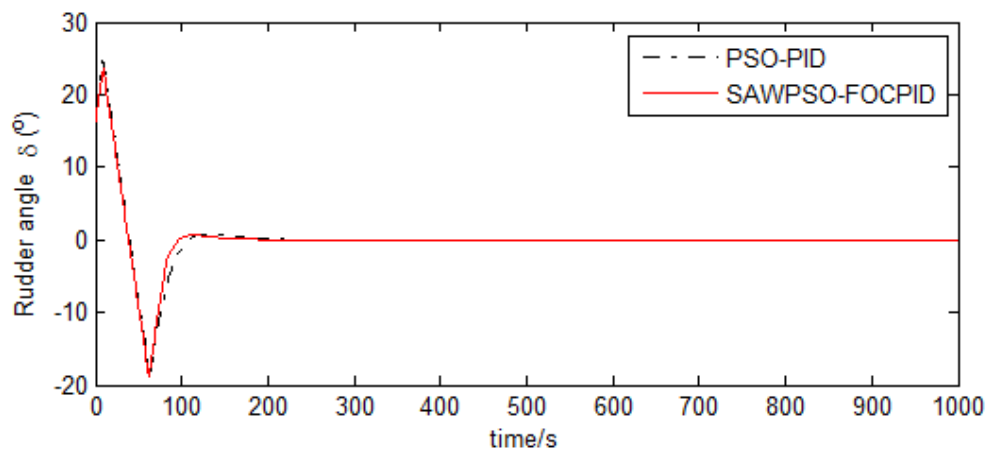

Figure 3. Ship Rudder Angle Output Curve under no Wind and Wave Disturbance 


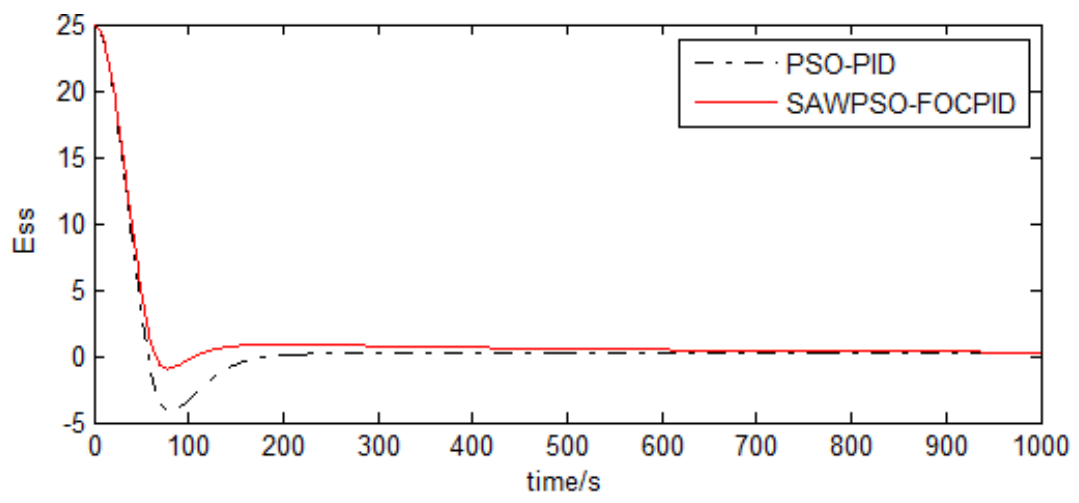

Figure 4. Ship Course Tracking Error Curve under no Wind and Wave Disturbance

Through the simulation, the controller and control effect of two different control methods are respectively shown in table 2 and 3 .

Table 2. Optimal Control Parameters of Two Different PID Controllers

\begin{tabular}{llllll}
\hline Controller type & $K_{P}$ & $K_{I}$ & $K_{D}$ & $\lambda$ & $\mu$ \\
\hline PSO-PID & 1.2186 & 0 & 68.472 & 0 & 0 \\
SAWPSO- & 16.38 & -3.79 & 44.95 & 0.28 & 0.87 \\
FOCPID & & & & & \\
\hline
\end{tabular}

Table 3. Control Effect of Two Different PID Controllers

\begin{tabular}{|c|c|c|c|c|}
\hline Controller type & $\begin{array}{l}\text { Adjust time } \\
t_{s}\end{array}$ & $\begin{array}{l}\text { Rise } \\
\text { time } t_{r}\end{array}$ & $M^{\text {Overshoot }}$ & $\begin{array}{r}\text { Static } \\
\text { error } E_{s s}\end{array}$ \\
\hline PSO-PID & 192 & 83 & $16.54 \%$ & 0.2916 \\
\hline $\begin{array}{l}\text { SAWPSO- } \\
\text { FOCPID }\end{array}$ & 106 & 79 & $3.78 \%$ & 0.0212 \\
\hline
\end{tabular}

Meanwhile, when the ship is sailing, the disturbance of wind and wave has become one of the major causes of the ship yaw. In order to validate the robustness of fractional-order $P I^{\lambda} D^{\mu}$ control systems, disturbance of wind and wave is used in this design. A simple simulation method is used, that is using white noise to drive a typical two order oscillation systems [20]. The transfer function of wave model in six level winds is

$$
h(s)=\frac{0.4198 s}{s^{2}+0.3638 s+0.3675}
$$

Under wind and wave disturbance, the step response curves of two different control methods are shown in Figure 5, Figure 6 and Figure 7.

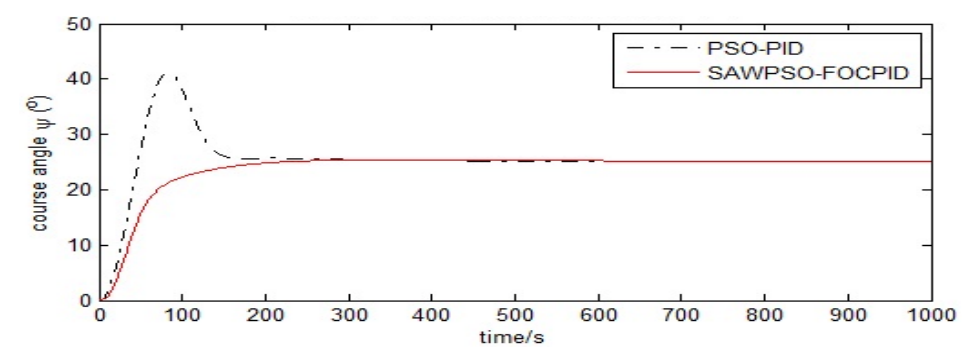

Figure 5. Ship Course Tracking Curve under Wind and Wave Disturbance 


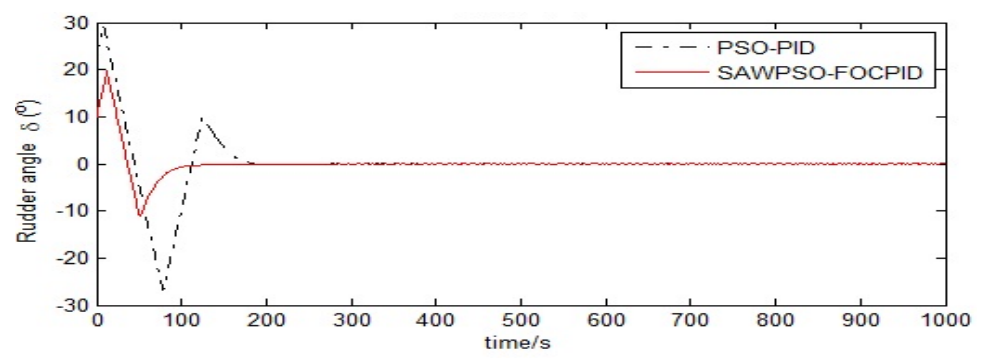

Figure 6. Ship Rudder Angle Output Curve under Wind and Wave Disturbance

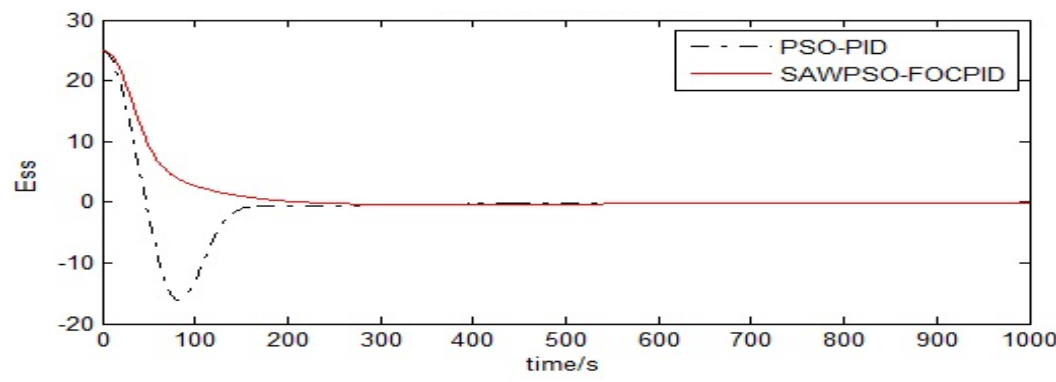

\section{Figure 7. Ship Course Tracking Error Curve under Wind and Wave Disturbance}

Through the simulation, the controller and control effect of two different control methods under wind and wave disturbance are respectively shown in table 4 and 5 .

Table 4. Optimal Control Parameters of Two Different PID Controllers

\begin{tabular}{llllll}
\hline $\begin{array}{c}\text { Controller } \\
\text { type }\end{array}$ & $K_{P}$ & $K_{I}$ & $K_{D}$ & $\lambda$ & $\mu$ \\
\hline PSO-PID & 22.81 & -3.77 & 36.77 & 0 & 0 \\
$\begin{array}{l}\text { SAWPSO- } \\
\text { OCPID }\end{array}$ & 10.04 & 4.73 & 45.15 & 0.275 & 0.891 \\
\hline
\end{tabular}

Table 5. Control Effect of Two Different PID Controllers

\begin{tabular}{lllll}
\hline Controller type & $\begin{array}{l}\text { Adjust time } \\
t_{s}\end{array}$ & Rise time $t_{r}$ & Overshoot $M$ & $\begin{array}{c}\text { Static } \\
\text { error } E_{s s}\end{array}$ \\
\hline PSO-PID & 248 & 86 & $68.08 \%$ & 0.1306 \\
SAWPSO-OCPID & 225 & 225 & $0 \%$ & 0.0271 \\
\hline
\end{tabular}

From Figure 2 to Figure7 and Table2 to Table5, we can see that the fractional-order $P I^{\lambda} D^{\mu}$ control based on SAWPSO algorithm can be faster to achieve stable and the control effect is better than integer-order PID controller based on PSO algorithm under the uncertainty of wind and waves interference. In the same interference conditions, the fractional-order $P I^{\lambda} D^{\mu}$ control based on SAWPSO algorithm has more immunity ability and the adaptive ability.

\section{Conclusions}

In this paper, according to the characteristics of USV, a design method for fractional-order control of USV course-keeping based on self-adaptive weight PSO algorithm was adopted. This method can solve the difficulties of tuning parameters 
more of fractional order controller and design complex, and further improve the dynamic and static performance of system. The simulation results show that the performance of SAWPSO-FOCPID controller is better than the conventional PSOPID controller. When the controller output is changed under the wind, wave disturbance and the control object is changed due to some factors, the SAWPSOFOCPID controller can overcome these effects. It has a high robustness, small overshoot and strong immunity. It has high spreading value in the area of ship PID automatic rudder.

\section{Acknowledgements}

The authors would like to thank all the reviewers for their constructive comments. This research was supported by the National Natural Science Foundation of China (Grant No.61374114, U1433124, 51475065), the Fundamental Research Funds for the Central Universities(DMU No.3132014321), Open Project Program of Provincial Key Laboratory for Computer Information Processing Technology, Soochow University (KJS1326), Open Project Program of Guangxi Key laboratory of hybrid computation and IC design analysis(HCIC201402), the Open Project Program of the Traction Power State Key Laboratory of Southwest Jiaotong University (TPL1403).

\section{References}

[1] Kolmanovsky and N.H. McClamroch, 'Developments in nonholonomic control problems', IEEE Control Systems Magazine, 15, pp.20-36 (1995).

[2] B. Fu and W.L. Luo, "Ship course control based on LSSVM-PID. Mechanical and Electrical Engineering”, 30(5), pp.632-635, (2013).

[3] [3] Y. Wang and C. Guo, "Adaptive control of ship course using a dynamic neural fuzzy algorithm", Journal of Harbin EngineeringUniversity, 30(10), pp.1159-1164, (2009).

[4] Podlubny, "Fractional-order System and Fractional-order Control", IEEE Transactions on Automatic Control, 44(1), pp.208-214, (1999).

[5] [5] I. Podlubny, "Realization of Fractional-order Control", Acta Montanistica Slovaca, 8 (4), pp.233235, (2003).

[6] C.J. Wu and Y.B. Zhang, "Vector control of three-phase voltage source PWM rectifier based on fractional-order controller", In Proceedings of the 26th Chinese Control and Decision Conference, (2014), CHINA, pp.2064-2069.

[7] F. Batlle, R. Perez and R. Castillo-García, "Simple fractional order controller combined with a Smith predictor for temperature control in a steel slab reheating furnace", International Journal of Control, Automation and Systems, 11( 3), pp.533-544, (2013).

[8] Choudhary and Kr. Santosh, "Stability and performance analysis of fractional order control systems", WSEAS Transactions on Systems and Control, 9( 1), pp.438-444, (2014).

[9] Ozdemir and Necati, "Fractional order control of fractional diffusion systems subject to input hysteresis", Journal of Computational and Nonlinear Dynamics, 5(2), pp.1-5,(2010).

[10] S.C. Yao and H.X. Pan, "A Fractional Order PID Controller for Synchronous Machine Excitation Using Particle Swarm Optimization”, Proceedings of the CSEE2010(2010), 30( 21), pp.91-97.

[11] B.T. Zhang and Y.G. Pi, "Integration of Fuzzy and Sliding Mode Control Based on Fractional Calculus Theory for Permanent Magnet Synchronous Motor, "Przeglad Elektrotchniczny, 87 (11), pp.251-255, (2011).

[12] W. Meng, C. Guo and Y. Liu, "Robust Adaptive Path Following for Underactuated Surface Vessels with Uncertain Dynamics", Marine Sci. Appl, 1, pp.244-250,(2012).

[13] X.L. Jia, X.K. Zhang, "Ship motion intelligent control and $\boldsymbol{H}_{\infty}$ robust control", China, Dalian Maritime University press, Dalian (2002)

[14] X.H. Wu, "Ship maneuverability and Seakeeping", China Communications Press, China Beijing (1999)

[15] Y.S. Li, C.N. Zhao and T.Lu, "Research and application of fractional order advanced control system", Beijing Normal University press, China Beijing (2012)

[16] J. KENNEDY and R. EBERHART, "Particle swarm optimization", In Proceedings of the IEEE International Conference on Neural Networks, (1995), Perth, Australia, pp.1942-1948.

[17] P.J. Angeline, "Using selection to improve particle swarm optimization", IEEE International Conference on Evolutionary Computation , (2007), Anchorage, Alaska, USA, pp.84-89.

[18] S. NAKA, T. GENJI, T. YURA, "A hybrid particle swarm optimization for distribution state estimation. IEEE Trans on Power Syst, 18( 1), pp.60-68, (2003). 
[19] Wonohadidjojo, M. Daniel, "Position control of electro-hydraulic actuator system using fuzzy logic controller optimized by particle swarm optimization", International Journal of Automation and Computing, 10( 3), pp.181-193, ( 2013).

[20] C.E. Yang, X.L. Jia and Y.J. Bi, "Ship rudder damping and robust control", China, Dalian Maritime University press, Dalian (2001)
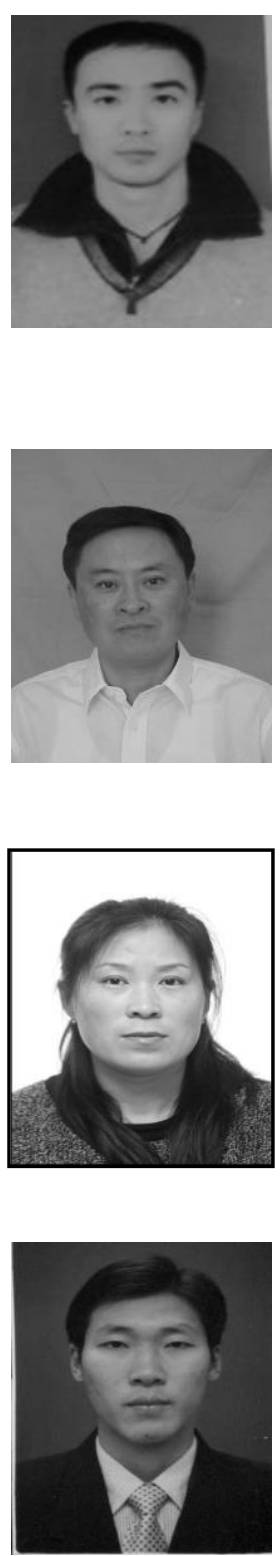

\section{Authors}

Li Guang-yu graduated from Dalian Jiaotong University, China in 2002. He received the M.S. degrees from school of electrical engineering college of Dalian Jiaotong University, Dalian, China, in 2005. He is pursuing Ph.D. degree at Navigation College of Dalian Maritime University, Dalian, China, in 2011.He is an assistant professor at Software College of Dalian Jiaotong University, China. His research interests include artificial intelligence, fractional-order control, particle swarm optimization algorithm and so on.

E-mail: ligyu@163.com

Guo Chen graduated from Chongqing University, China in 1982. He received his M. Sc. degree from University in 1985 and his Ph.D. degree from Dalian Maritime University, China in 1991, respectively.

$\mathrm{He}$ is currently a professor at College of Information science and technology, Dalian Maritime University. His research interests include adaptive control, intelligent control, ship engine simulation system, and virtual reality technology.

E-mail:dmuguoc@126.com

Li Yan-xin graduated from Dalian Jiaotong University, China in 2002. She received the M.S. degrees from school of electrical engineering college of Dalian Jiaotong University, Dalian, China, in 2005. She is an assistant professor at Software College of Dalian Jiaotong University, China. Her research interests include artificial intelligence, neural network, rough set theory and so on.

E-mail:lyx7977@163.com

Deng Wu graduated from Dalian Jiaotong University, China in 2001. He received the M.S. degrees from school of electrical engineering college of Dalian Jiaotong University, Dalian, China, in 2006. He received the Ph.D. degree at Information and Technology College of Dalian Maritime University, Dalian, China, in 2012. He is an assistant professor at Software College of Dalian Jiaotong University, China. His research interests are intelligent optimization and information processing, intelligent fault diagnosis.

E-mail:dw7689@gmail.com 